\title{
Pregnancy plasma glucose levels exceeding the American Diabetes Association thresholds, but below the National Diabetes Data Group thresholds for gestational diabetes mellitus, are related to the risk of neonatal macrosomia, hypoglycaemia and hyperbilirubinaemia
}

\author{
A. Ferrara • N. S. Weiss • M. M. Hedderson • \\ C. P. Quesenberry Jr. • J. V. Selby • I. J. Ergas • T Peng • \\ G. J. Escobar • D. J. Pettitt • D. A. Sacks
}

Received: 20 July 2006 / Accepted: 2 October 2006 / Published online: 14 November 2006

(C) Springer-Verlag 2006

\begin{abstract}
Aims/hypothesis Gestational diabetes mellitus (GDM) is a risk factor for perinatal complications. In several countries, the criteria for the diagnosis of GDM have been in flux, the American Diabetes Association (ADA) thresholds recommended in 2000 being lower than those of the National Diabetes Data Group (NDDG) that have been in use since 1979. We sought to determine the extent to which infants of women meeting only the ADA criteria for GDM are at increased risk of neonatal complications.

Materials and methods In a multiethnic cohort of 45,245 women who did not meet the NDDG criteria and were not treated for GDM, we conducted nested case-control
\end{abstract}

A. Ferrara $(\square) \cdot$ M. M. Hedderson $\cdot$ C. P. Quesenberry Jr.

J. V. Selby $\cdot$ I. J. Ergas $\cdot$ T. Peng $\cdot$ G. J. Escobar

Division of Research, Kaiser Permanente Medical Care

Program of Northern California,

2000 Broadway,

Oakland, CA 94612, USA

e-mail: Assiamira.Ferrara@kp.org

A. Ferrara $\cdot$ N. S. Weiss

Department of Epidemiology, School of Public Health

and Community Medicine, University of Washington,

Seattle, WA, USA

D. J. Pettitt

Sansum Diabetes Research Institute,

Santa Barbara, CA, USA

D. A. Sacks

Kaiser Foundation Hospital,

Bellflower, CA, USA studies of three complications of GDM that occurred in their infants: macrosomia (birthweight $>4,500 \mathrm{~g}, n=494$ ); hypoglycaemia (plasma glucose $<2.2 \mathrm{mmo} / \mathrm{l}, n=488$ ); and hyperbilirubinaemia (serum bilirubin $\geq 342 \mu \mathrm{mol} / 1(20 \mathrm{mg} / \mathrm{dl})$, $n=578$ ). We compared prenatal glucose levels of the mothers of these infants and mothers of 884 control infants. Results Women with GDM by ADA criteria only (two or more glucose values exceeding the threshold) had an increased risk of having an infant with macrosomia (odds ratio $\mathrm{OR}=3.40,95 \% \mathrm{CI}=1.55-7.43$ ), hypoglycaemia $(\mathrm{OR}=2.61,95 \% \mathrm{CI}=0.99-6.92)$ or hyperbilirubinaemia $(\mathrm{OR}=2.22,95 \% \mathrm{CI}=0.98-5.04)$. Glucose levels $1 \mathrm{~h}$ after the 100-g glucose challenge that exceeded the ADA threshold were particularly strongly associated with each complication.

Conclusions/interpretation These results lend support to the ADA recommendations and highlight the importance of the 1-h glucose measurement in a diagnostic test for GDM.

Keywords Clinical science and care - Complications . Epidemiology $\cdot$ Human $\cdot$ Hypoglycaemia $\cdot$ Pregnancy
Abbreviations
ADA American Diabetes Association
GCT glucose challenge test
GDM gestational diabetes mellitus
KP Kaiser Permanente Medical Care Program
NDDG National Diabetes Data Group
OR odds ratio 


\section{Introduction}

Gestational diabetes mellitus (GDM) is associated with increased risk of adverse perinatal outcomes [1, 2]. Its clinical recognition and treatment may reduce these risks [3]. In several countries the $100-\mathrm{g}, 3-\mathrm{h}$ OGTT is used to diagnose GDM. The diagnostic glucose thresholds using the 100-g, 3-h OGTT were first published by O'Sullivan and Mahan [4] in 1964 and were based on their ability to predict maternal diabetes subsequent to pregnancy. In this study the medium assayed was whole blood, and the laboratory method used (Somogyi-Nelson) assayed for all reducing substances including glucose. In 1979, the National Diabetes Data Group (NDDG) [5] proposed glucose thresholds higher than those proposed by O'Sullivan and Mahan, based on a conversion from whole blood to plasma glucose, but not considering differences in assay method. In 1982, Carpenter and Coustan [6] further modified the O'Sullivan and Mahan thresholds by including a correction from the assay for reducing substances to those specific for glucose. A study conducted by Sacks et al. [7] confirmed the validity of the correction proposed by Carpenter and Coustan. In 1998 the Fourth International Workshop Conference on GDM [8] proposed that the Carpenter and Coustan thresholds [6] be used for the interpretation of the $100 \mathrm{~g}$, 3-h OGTT. In 2000, the American Diabetes Association (ADA) [9] endorsed the recommendations of the Fourth International Workshop Conference [8]. The newly recommended ADA thresholds [9] are lower than those of the NDDG [5] (Table 1). The scientific rationale for revising the glucose thresholds downward included limited data suggesting the possibility that maternal glucose levels lower than the NDDG thresholds [5] may be associated with increased risk of neonatal macrosomia, pre-eclampsia and Caesarean section [8]. No studies have had a large enough sample size to assess whether glucose levels lower than the NDDG thresholds [5] are associated with other less common neonatal complications, such as hypoglycaemia and hyperbilirubinaemia, which have been previously found to be related to glucose levels above the NDDG thresholds [10].

Table 1 OGTT threshold values (glucose, mmol/1 (mg/dl)) for diagnosis of GDM

\begin{tabular}{lll}
\hline & NDDG criteria $[5]^{\mathrm{a}}$ & ADA criteria $[9]^{\mathrm{a}}$ \\
\hline Fasting & $5.8(105)$ & $5.3(95)$ \\
$1-\mathrm{h}$ & $10.5(190)$ & $10.1(180)$ \\
$2-\mathrm{h}$ & $9.1(165)$ & $8.7(155)$ \\
$3-\mathrm{h}$ & $8.0(145)$ & $7.8(140)$ \\
\hline
\end{tabular}

${ }^{a}$ By both criteria, gestational diabetes is defined as having at least two plasma glucose measurements during the diagnostic test at the reported cutoff points or higher.
The purpose of this study was to investigate whether, among women who did not meet the NDDG criteria [5] for GDM, the risk of neonatal macrosomia, hypoglycaemia and hyperbilirubinaemia increased with increasing levels of maternal glycaemia, and specifically whether the risks of these complications were increased in women with GDM by ADA criteria [9] only. The objectives were addressed by conducting nested case-control studies within a multiethnic cohort of 45,245 women who delivered singletons at the Kaiser Permanente Medical Care Program (KP hereafter) Northern California, did not meet the NDDG criteria [5] and were not treated for GDM. We first present the unadjusted odds ratios (ORs) relating maternal plasma glucose levels to neonatal outcomes because, in the clinical setting, a threshold for action would be set that typically would not consider other patient characteristics. Next, we explored whether the associations that we observed were explained by possible confounders.

\section{Subjects and methods}

Cohort definition and identification KP Northern California is a group-practice, prepaid health plan that includes 16 hospitals. The KP provides comprehensive medical services to approximately 3.0 million members (approximately 30\% of the general population in the geographic areas covered). The sociodemographic characteristics of KP membership closely approximate the general population ethnically and socioeconomically, except for the extremes of the income distribution $[11,12]$.

We searched the KP hospital discharge and billing claim databases to identify all singleton live births, and searched the KP laboratory database to obtain all plasma glucose values measured during screening glucose challenge tests (GCTs) and diagnostic 3-h OGTTs [13]. We restricted our cohort to women who delivered between 1 January 1996 and 31 June 1998, because the recommendations from the 4th International Workshop Conference on GDM [8] were published in August 1998. Thereafter, the clinical management of women meeting the ADA thresholds [9] for GDM diagnosis might have changed, and the association between these glucose thresholds and adverse neonatal outcomes could have been obscured. During this 30-month period, $94 \%$ of all women who delivered a live infant or had a stillbirth had been screened for GDM with a 50-g, 1-h oral GCT during the second trimester. If abnormal (1-h plasma glucose levels $\geq 7.8 \mathrm{mmol} / 1(140 \mathrm{mg} / \mathrm{dl}))$, this test was followed by a diagnostic $100-\mathrm{g}$, 3-h OGTT performed in the morning after a 12-h fast [9]. Before the diagnostic test, women were mailed multilingual instructions to ingest a minimum of $150 \mathrm{~g}$ of carbohydrate per day for 3 days before testing. We identified 72,946 pregnancies without 
recognised pre-existing diabetes who delivered a singleton live infant. After excluding 4,560 pregnancies who were not screened by the 50-g, 1-h GCT we then excluded 21,297 pregnancies who had the screening 50-g, 1-h GCT before 24 weeks of gestational age or after 28 weeks of gestational age. In order to exclude the confounding effect of treatment on the association between maternal glycaemia and neonatal complications we then excluded 362 women who had a history of GDM and 1,482 pregnancies with plasma glucose results during the index pregnancy that met the NDDG criteria [5] for GDM diagnosis, leaving 45,245 eligible women for the selection of cases and controls (Fig. 1). Of these 45,245 pregnancies, there were 811 whose 100-g OGTT results met the ADA criteria [9] only.

Case selection, definition and confirmation We searched the hospital databases and the database of the regional laboratory (where all tests are performed) to identify newborns who had the following neonatal complications: (1) macrosomia (birthweight $>4,500 \mathrm{~g}, n=1,073$ ); (2) hypoglycaemia (at least one plasma glucose $<2.2 \mathrm{mmol} / 1$

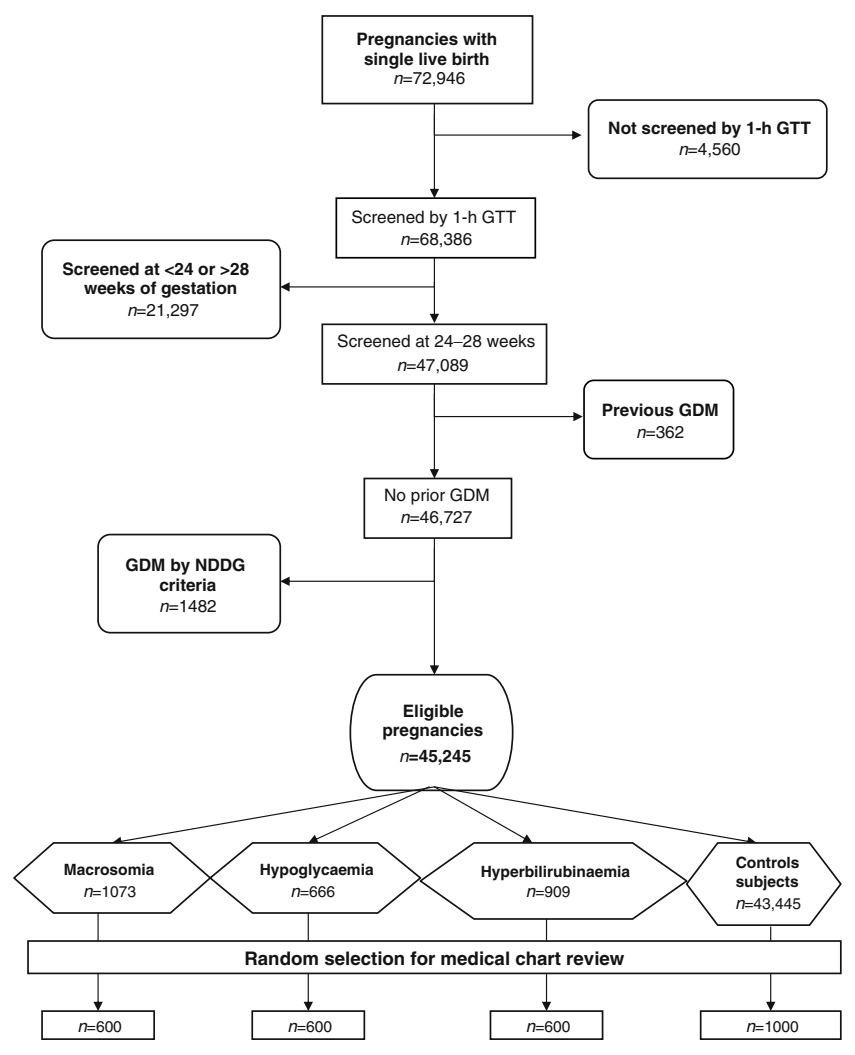

Fig. 1 Study population exclusion criteria and selection of motherinfant pairs with and without complications among women without recognised pre-existing diabetes who delivered at the KP Northern California in January 1996 to June 1998. Macrosomia is infant birthweight $>4,500 \mathrm{~g}$; hypoglycaemia is at least one plasma glucose value $<2.2 \mathrm{mmol} / 1$ during the first 3 days of life; hyperbilirubinaemia is at least one total serum bilirubin concentration $\geq 342 \mu \mathrm{mol} / 1$ was identified during the first 30 days of life
(40 mg/dl), $n=666$ ); or (3) hyperbilirubinaemia (at least one total serum bilirubin greater $\geq 342 \mu \mathrm{mol} / 1(20 \mathrm{mg} / \mathrm{dl})$, $n=909$ ). For each case group, a random sample of 600 infants was selected without knowledge of the maternal glucose values at the screening and the diagnostic test. Trained abstractors reviewed the medical records of mother-infant pairs that were randomly selected and confirmed eligibility criteria and case definitions for the following: 488 (out of 505) with macrosomic infants, 486 (out of 498) with infants with hypoglycaemia and 578 (out of 588) with infants with hyperbilirubinaemia. Macrosomia was confirmed if the infant had a birthweight $>4,500 \mathrm{~g}$ and did not have fetal hydrops. Hypoglycaemia was confirmed if a laboratory test showed a plasma glucose value $<2.2 \mathrm{mmol} / \mathrm{l}$ during the first 3 days of life. Hyperbilirubinaemia was confirmed if at least one total serum concentration $\geq 342 \mu \mathrm{mol} / 1$ was identified during the first 30 days of life and the following conditions were not present: positive direct antiglobulin test, glucose-6-phosphate dehydrogenase deficiency, ABO incompatibility or Rhesus alloisoimmunisation.

Control selection From the remaining 43,445 women in this cohort, a random sample of 1,000 infants was selected without knowledge of the maternal glucose values. We reviewed the medical records of 960 mother-infant pairs who were randomly selected as controls and confirmed eligibility for 879 of them. If a potential control infant met the criteria for one of the three case conditions the motherinfant pair was excluded from that particular analysis, but the pair would have been included as a control pair in the other case control analyses. Among the infants who were selected as controls there were seven with macrosomia, one with hypoglycaemia and three with hyperbilirubinaemia, according to our case definitions. In addition, we excluded 26 controls with a capillary glucose value $<2.2 \mathrm{mmol} / 1$ (assessed in the hospital by a capillary glucose test strip and not followed by a laboratory measurement) from the analysis of hypoglycaemia because they may have met the case definition for hypoglycaemia if a laboratory test had been performed.

Data collection Medical chart review was conducted by trained abstractors blinded to case/control status to ascertain that criteria for inclusion (screening 50-g GCT performed at 24-28 weeks of gestation according to the earliest ultrasound performed prior to 24 weeks of gestation) were met and whether any of the exclusion criteria (history of diabetes or GDM, 100-g OGTT plasma glucose results diagnostic of GDM according to the NDDG criteria [5] or multiple births during the index pregnancy) were present. In addition, women were excluded if a physician diagnosis of GDM, ADA diet or insulin prescription had been noted 
in the medical chart at the first clinic visit after the screening or the diagnostic test despite the glucose values below the NDDG thresholds [5] $(n=25)$, but were included $(n=1)$ if any of the above had been noted later. Other information abstracted included any plasma glucose test performed during pregnancy, age at delivery, gestational age and weight at delivery, education, marital status, prepregnancy weight, height, parity, family history of diabetes, smoking, medication use, blood pressure and history of hypertension. Self-reported maternal race-ethnicity was collected from the infants' birth certificates found in the charts. Pre-pregnancy weight was based on the mother's self-reported pre-pregnancy weight recorded on the prenatal form at her first prenatal visit. For the $25 \%$ of women for whom these data were not available, the measured weight recorded in the chart prior to her last menstrual period was used. To examine agreement between the two methods of estimating pre-pregnancy weight, we compared selfreported pre-pregnancy weight and a weight measured of the 695 women for whom both data were available. The Pearson correlation coefficient between the two weights was 0.97 and the mean self-reported weight was $1.6 \mathrm{~kg}$ less than the measured weight. Pre-pregnancy BMI was calculated as pre-pregnancy weight $(\mathrm{kg})$ divided by height squared $\left(\mathrm{m}^{2}\right)$. Rate of maternal pregnancy weight gain was calculated as total pregnancy weight gain minus infant birthweight divided by weeks of gestation when the last weight was measured (e.g. within 2 weeks before delivery). Hypertension before or at the date of the screening test was defined as present if a woman had a physician diagnosis, or was using antihypertensive medications or had systolic blood pressure $\geq 130 \mathrm{mmHg}$, or diastolic blood pressure $\geq 90 \mathrm{mmHg}$ on or before the screening date.

Definitions of maternal glycaemia variables All plasma glucose measurements were performed using the hexokinase method by the regional laboratory of KP Northern California. This laboratory participates in the College of American Pathologists accreditation and monitoring programme. All pregnancy glycaemia variables for our analyses were dichotomised according to the ADA thresholds [9] (Table 1).

Statistical analysis Unconditional logistic regression was used to obtain ORs as estimates of the relative risk of each infant complication (macrosomia, hypoglycaemia and hyperbilirubinaemia) associated with maternal glycaemia. Two analytic approaches were used to examine maternal glycaemia variables. In each analysis, women with normal plasma glucose screening values measured $1 \mathrm{~h}$ after the 50 -g GCT $<7.8 \mathrm{~mol} / 1$ formed the reference category. In the first analysis, the association between increasing number of glucose values obtained during the diagnostic test meeting the ADA thresholds [9] and each neonatal complication was assessed. Women with abnormal screening values measured $1 \mathrm{~h}$ after the 50 -g GCT $(\geq 7.8 \mathrm{mmol} / \mathrm{l})$ were placed into one of three categories: (1) all glucose values obtained during the diagnostic 100-g OGTT below the ADA thresholds; or (2) only one glucose value during the diagnostic test meeting or exceeding the ADA thresholds; or (3) at least two glucose values obtained during the diagnostic test meeting or exceeding the ADA thresholds. The second set of analyses looked separately at each glucose measurement obtained during the diagnostic test (fasting, 1-, 2- and 3-h) according to the ADA thresholds. For example, when fasting plasma glucose was analysed, women with abnormal screening were placed into one of two categories: (1) fasting glucose values below the ADA threshold $(<5.3 \mathrm{mmol} / \mathrm{l})$; or (2) fasting glucose values that equalled or exceeded the ADA threshold $(\geq 5.3 \mathrm{mmol} / \mathrm{l})$.

We first present unadjusted ORs relating maternal plasma glucose levels to neonatal outcomes. Next, we adjusted all models for maternal age at delivery and race-ethnicity. The other potentially confounding variables entered into the model individually as covariates were pre-pregnancy BMI, education, marital status, parity, family history of diabetes, hypertension prior to screening and smoking. For each neonatal complication, only maternal pre-pregnancy BMI changed the OR by $10 \%$ or more. The latter was added to the final adjusted model along with age and race-ethnicity. We also examined as potential confounders gestational age at delivery and rate of pregnancy weight gain. We examined the potential interaction between each of the maternal glycaemia variables and race-ethnicity (nonHispanic white women vs women from other race-ethnicity groups), age ( $<30 \mathrm{vs} \geq 30$ years), and pre-pregnancy BMI $\left(<25.0 \mathrm{vs} \geq 25.0 \mathrm{~kg} / \mathrm{m}^{2}\right)$ in predicting the three neonatal outcomes.

This study was approved by the human subjects committee of the Kaiser Foundation Research Institute.

\section{Results}

Table 2 compares characteristics of mothers of infants with complications with the characteristics of mothers of control infants. Relative to mothers of control infants, mothers of macrosomic infants were more likely to be non-Hispanic white, whereas mothers of infants with hypoglycaemia were more likely to be Hispanic, and mothers of infants with hyperbilirubinaemia were more likely to be Asian. Mothers of macrosomic infants were older, less likely to be primiparous and more likely to deliver at 41 or more weeks of gestation than control mothers, whereas mothers of hypoglycaemic and hyperbilirubinaemic infants were 
more likely to be primiparous and more likely to deliver at $<37$ weeks of gestation. As compared with mothers of controls, mothers of infants with macrosomia and hypoglycaemia were more likely to be obese prior to pregnancy and to have hypertension prior to the screening test for GDM. Mothers of infants with hyperbilirubinaemia tended to be more highly educated than mothers of control infants.
Tables 3 and 4 compare levels of plasma glucose during the screening 50-g GCT and the diagnostic 100-g OGTT among cases and controls. In unadjusted analyses the risk of neonatal macrosomia, hypoglycaemia and hyperbilirubinaemia increased with increasing number of glucose values obtained during the 100-g OGTT that met or exceed the ADA thresholds [9]. Compared with normal screening $(\mathrm{OR}=1.00)$, in women with abnormal screening the ORs increased across levels of maternal glycaemia (all values
Table 2 Characteristics of mothers who did not meet the NDDG criteria by case-control status: KP Northern California, January 1996-June 1998
Macrosomia is infant birthweight $>4,500 \mathrm{~g}$ and no fetal hydrops; hypoglycaemia is at least one plasma glucose value $<2.2 \mathrm{mmol} / 1$ during the first 3 days of life; hyperbilirubinaemia is at least one total serum bilirubin concentration $\geq 342 \mu \mathrm{mol} / 1$ was identified during the first 30 days of life and the following conditions were not present: positive direct antiglobulin test, glucose-6-phosphate dehydrogenase deficiency, ABO incompatibility or Rhesus alloisoimmunisation.

Values are percentages or means \pm SD.

${ }^{a}$ Since we exclude controls who meet the case definitions in the analyses, this is the not the exact number of controls used in the models presented in Table 4.

\begin{tabular}{|c|c|c|c|c|}
\hline & $\begin{array}{l}\text { Controls } \\
(n=879)^{\mathrm{a}}\end{array}$ & $\begin{array}{l}\text { Macrosomia } \\
(n=488)\end{array}$ & $\begin{array}{l}\text { Hypoglycaemia } \\
(n=486)\end{array}$ & $\begin{array}{l}\text { Hyperbilirubinaemia } \\
(n=578)\end{array}$ \\
\hline \multicolumn{5}{|l|}{ Age (years) } \\
\hline Means \pm SD & $29.3 \pm 5.9$ & $30.2 \pm 5.6$ & $29.2 \pm 6.3$ & $29.6 \pm 5.6$ \\
\hline$<25$ & 23.1 & 16.8 & 26.1 & 18.0 \\
\hline $25-29$ & 24.5 & 27.9 & 25.1 & 29.2 \\
\hline $30-34$ & 33.3 & 30.9 & 26.1 & 33.2 \\
\hline$\geq 35$ & 19.1 & 24.4 & 22.7 & 19.6 \\
\hline \multicolumn{5}{|l|}{ Race-ethnicity } \\
\hline Non-Hispanic white & 52.0 & 66.4 & 45.3 & 42.0 \\
\hline African-American & 9.7 & 5.5 & 10.9 & 4.3 \\
\hline Asian & 9.2 & 3.9 & 6.8 & 20.8 \\
\hline Hispanic & 17.3 & 15.0 & 22.8 & 16.4 \\
\hline Other & 10.9 & 8.2 & 13.8 & 15.6 \\
\hline Unknown & 0.9 & 1.0 & 0.4 & 0.9 \\
\hline \multicolumn{5}{|l|}{ Education (years) } \\
\hline$<12$ & 11.7 & 7.8 & 11.1 & 8.0 \\
\hline 12 & 29.4 & 31.0 & 32.8 & 25.8 \\
\hline $13-15$ & 28.6 & 30.1 & 29.4 & 27.9 \\
\hline$\geq 16$ & 28.6 & 29.7 & 25.5 & 37.2 \\
\hline Unknown & 1.7 & 1.4 & 1.2 & 1.1 \\
\hline Married & 75.1 & 81.4 & 76.3 & 81.0 \\
\hline \multicolumn{5}{|c|}{ Pre-pregnancy BMI $\left(\mathrm{kg} / \mathrm{m}^{2}\right)$} \\
\hline Means \pm SD & $24.4 \pm 5.0$ & $26.4 \pm 5.5$ & $25.4 \pm 5.7$ & $24.3 \pm 5.0$ \\
\hline$<20.0$ & 13.4 & 5.5 & 10.1 & 14.4 \\
\hline $20.0-24.9$ & 43.5 & 35.1 & 38.5 & 45.7 \\
\hline $25.0-29.9$ & 21.1 & 27.2 & 23.3 & 18.0 \\
\hline$\geq 30.0$ & 11.5 & 21.3 & 14.4 & 12.1 \\
\hline Unknown & 10.5 & 10.9 & 13.7 & 9.8 \\
\hline \multicolumn{5}{|c|}{ Family history of diabetes } \\
\hline First degree & 11.6 & 12.5 & 13.6 & 10.6 \\
\hline Second/third degree & 21.0 & 21.3 & 18.7 & 20.9 \\
\hline No history & 59.3 & 56.6 & 61.5 & 60.0 \\
\hline Unknown & 8.1 & 9.6 & 6.2 & 8.5 \\
\hline Hypertension & 29.7 & 40.2 & 37.7 & 32.2 \\
\hline Primiparous & 42.7 & 30.5 & 51.2 & 61.2 \\
\hline \multicolumn{5}{|c|}{ Smoked during pregnancy } \\
\hline No & 89.9 & 88.8 & 90.3 & 92.7 \\
\hline Yes, but quit & 4.2 & 4.9 & 2.5 & 4.7 \\
\hline Yes, continued & 5.3 & 4.7 & 7.2 & 1.2 \\
\hline Unknown & 0.6 & 1.6 & 0.0 & 1.4 \\
\hline \multicolumn{5}{|c|}{ Gestational age at delivery (weeks) } \\
\hline$<37$ & 2.8 & 0.2 & 24.1 & 9.8 \\
\hline $37-40$ & 65.0 & 34.8 & 54.3 & 77.0 \\
\hline$\geq 41$ & 32.2 & 65.0 & 21.6 & 13.2 \\
\hline
\end{tabular}


during the 100-g OGTT below the ADA thresholds [9], only one value meeting or exceeding the ADA thresholds [9], and at least two values meeting or exceeding the ADA thresholds [9]) for macrosomia ( $p$ trend $=0.0002$ ), hypoglycaemia ( $p$ trend $=0.03$ ) and hyperbilirubinaemia ( $p$ trend $=0.007$ ). Women who had GDM by ADA criteria only were three times more likely to have an infant with macrosomia and approximately two times more likely to have an infant with hypoglycaemia or hyperbilirubinaemia, although the $95 \%$ CI around the OR for hypoglycaemia and hyperbilirubinaemia included one. In models adjusted for age, race-ethnicity and pre-pregnancy BMI, all of the above associations were less strong.

A 1-h glucose value meeting or exceeding the ADA thresholds [9] was the glucose measurement most strongly associated with increased risk of each neonatal complication, before and after adjusting for maternal age, race-ethnicity and pre-pregnancy BMI (Table 4). A fasting glucose value meeting or exceeding the ADA thresholds [9] was strongly associated with macrosomia before and after adjustments, but not with hypoglycaemia and hyperbilirubinaemia.

Adding gestational age to the models adjusted for age, race-ethnicity and pre-pregnancy BMI did not materially change the observed associations between the ADA glucose thresholds and neonatal complications. The ORs (95\% CI) associated with two or more glucose values meeting or exceeding the ADA thresholds [9] were 3.27 (1.44-7.45) for macrosomia, 2.75 (1.01-7.52) for hypoglycaemia, and 1.68 (0.71-4.01) for hyperbilirubinaemia.

In analyses conducted among a subgroup of women with data on pregnancy weight gain (731 mothers of control infants, 413 mothers of macrosomic infants, 388 mothers of hypoglycaemic infants and 420 mothers of hyperbilirubinaemic infants) adding rate of weight gain to the models adjusted for age, race-ethnicity and prepregnancy BMI did not materially change the observed associations between the ADA glucose thresholds and neonatal complications. The OR $(95 \% \mathrm{CI})$ associated with two or more glucose values meeting or exceeding the ADA thresholds [9] was 3.44 (1.26-9.42) for macrosomia, 2.75 (0.94-8.08) for hypoglycaemia and $2.70(0.92-7.92)$ for hyperbilirubinaemia.

The size of the association between maternal hyperglycaemia and the three neonatal complications differed little according to maternal age or pre-pregnancy BMI. For macrosomia and hyperbilirubinaemia (but not hypoglycaemia), there was a suggestion of a stronger association

Table 3 Performance on 50-g oral GCT and 100-g OGTT in mothers who did not meet the NDDG criteria by case/control status: KP Northern California, January 1996-June 1998

\begin{tabular}{|c|c|c|c|c|}
\hline Plasma glucose status & $\begin{array}{l}\text { Controls } \\
(n=879)^{\mathrm{a}}\end{array}$ & $\begin{array}{l}\text { Macrosomia } \\
(n=488)\end{array}$ & $\begin{array}{l}\text { Hypoglycaemia } \\
(n=486)\end{array}$ & $\begin{array}{l}\text { Hyperbilirubinaemia } \\
(n=578)\end{array}$ \\
\hline Normal 1-h GCT & 86.7 & 81.9 & 83.1 & 82.9 \\
\hline Abnormal 1-h GCT & 13.3 & 18.1 & 16.9 & 17.1 \\
\hline \multicolumn{5}{|c|}{ Number of values exceeding ADA thresholds during the 3-h OGTT } \\
\hline 0 & 9.1 & 7.8 & 10.7 & 9.5 \\
\hline 1 & 3.1 & 6.6 & 4.1 & 5.2 \\
\hline$\geq 2$ & 1.1 & 3.7 & 2.1 & 2.4 \\
\hline \multicolumn{5}{|c|}{ Fasting plasma glucose (mmol/1) } \\
\hline$<5.3$ & 12.3 & 14.4 & 15.3 & 15.9 \\
\hline$\geq 5.3^{\mathrm{b}}$ & 1.0 & 3.7 & 1.6 & 1.2 \\
\hline \multicolumn{5}{|c|}{ 1-h plasma glucose $(\mathrm{mmol} / \mathrm{l})$} \\
\hline$<10.1$ & 11.9 & 12.6 & 13.6 & 13.3 \\
\hline$\geq 10.1^{\mathrm{b}}$ & 1.4 & 5.5 & 3.3 & 3.8 \\
\hline \multicolumn{5}{|c|}{ 2-h plasma glucose $(\mathrm{mmol} / \mathrm{l})$} \\
\hline$<8.7$ & 11.3 & 14.4 & 14.4 & 14.3 \\
\hline$\geq 8.7^{\mathrm{b}}$ & 2.0 & 3.7 & 2.5 & 2.6 \\
\hline \multicolumn{5}{|c|}{ 3-h plasma glucose $(\mathrm{mmol} / \mathrm{l})$} \\
\hline$<7.8$ & 12.4 & 16.3 & 15.7 & 14.9 \\
\hline$\geq 7.8^{\mathrm{b}}$ & 0.9 & 1.8 & 1.2 & 2.2 \\
\hline
\end{tabular}

Macrosomia is infant birthweight $>4,500 \mathrm{~g}$ and no fetal hydrops; hypoglycaemia is at least one plasma glucose value $<2.2 \mathrm{mmol} / 1 \mathrm{during}$ the first 3 days of life; hyperbilirubinaemia is at least one total serum bilirubin concentration $\geq 342 \mu \mathrm{mol} / 1$ was identified during the first 30 days of life and the following conditions were not present: positive direct antiglobulin test, glucose-6-phosphate dehydrogenase deficiency, $\mathrm{ABO}$ incompatibility or Rhesus alloisoimmunisation.

Values are percentages.

${ }^{\text {a }}$ Since we exclude controls who meet the case definitions in the analyses, this is the not the exact number of controls used in the models presented in Table 4.

${ }^{\mathrm{b}}$ ADA threshold for that time period 
Table 4 Odds ratios and 95\% CI for neonatal complications associated with performance on 50-g oral GCT and 100-g OGTT among women who did not meet the NDDG criteria: KP Northern California, January 1996-June 1998

\begin{tabular}{|c|c|c|c|c|c|c|}
\hline \multirow[t]{2}{*}{ Plasma glucose status } & \multicolumn{2}{|c|}{ Macrosomia $(n=488)$} & \multicolumn{2}{|c|}{ Hypoglycaemia $(n=486)$} & \multicolumn{2}{|c|}{ Hyperbilirubinaemia $(n=578)$} \\
\hline & Unadjusted & Adjusted $^{\mathrm{a}}$ & Unadjusted & Adjusted $^{\mathrm{a}}$ & Unadjusted & Adjusted $^{\mathrm{a}}$ \\
\hline Normal 1-h GCT & 1.00 & 1.00 & 1.00 & 1.00 & 1.00 & 1.00 \\
\hline \multicolumn{7}{|c|}{ Number of values exceeding ADA thresholds during the 3-h OGTT } \\
\hline 0 & $0.91(0.61-1.36)$ & $0.86(0.57-1.31)$ & $1.20(0.83-1.74)$ & $1.23(0.85-1.80)$ & $1.10(0.77-1.59)$ & $0.97(0.67-1.41)$ \\
\hline 1 & $2.24(1.32-3.79)$ & $1.99(1.15-3.45)$ & $1.35(0.75-2.45)$ & $1.27(0.70-2.33)$ & $1.76(1.04-3.00)$ & $1.57(0.90-2.72)$ \\
\hline$\geq 2$ & $3.40(1.55-7.43)$ & $2.68(1.20-6.00)$ & $2.61(0.99-6.92)$ & $2.45(0.91-6.58)$ & $2.22(0.98-5.04)$ & $1.96(0.84-4.57)$ \\
\hline \multicolumn{7}{|c|}{ Fasting plasma glucose $(\mathrm{mmol} / \mathrm{l})$} \\
\hline$<5.3$ & $1.24(0.89-1.71)$ & $1.12(0.80-1.58)$ & $1.29(0.93-1.78)$ & $1.30(0.93-1.80)$ & $1.36(1.01-1.84)$ & $1.19(0.87-1.63)$ \\
\hline$\geq 5.3^{\mathrm{b}}$ & $3.78(1.68-8.48)$ & $3.18(1.38-7.31)$ & $1.83(0.68-4.91)$ & $1.60(0.59-4.35)$ & $1.23(0.46-3.34)$ & $1.16(0.42-3.26)$ \\
\hline \multicolumn{7}{|c|}{ 1-h plasma glucose (mmol/l) } \\
\hline$<10.1$ & $1.11(0.79-1.56)$ & $1.00(0.70-1.42)$ & $1.17(0.84-1.63)$ & $1.17(0.83-1.64)$ & $1.17(0.86-1.61)$ & $1.04(0.75-1.44)$ \\
\hline$\geq 10.1^{\mathrm{b}}$ & $4.25(2.13-8.48)$ & $3.84(1.88-7.84)$ & $2.93(1.32-6.51)$ & $2.93(1.31-6.59)$ & $2.91(1.43-5.93)$ & $2.56(1.23-5.32)$ \\
\hline \multicolumn{7}{|c|}{ 2-h plasma glucose $(\mathrm{mmol} / \mathrm{l})$} \\
\hline$<8.7$ & $1.35(0.97-1.88)$ & $1.26(0.89-1.78)$ & $1.31(0.94-1.82)$ & $1.32(0.94-1.84)$ & $1.33(0.97-1.82)$ & $1.17(0.85-1.63)$ \\
\hline$\geq 8.7^{\mathrm{b}}$ & $1.89(0.97-3.67)$ & $1.43(0.72-2.86)$ & $1.46(0.68-3.16)$ & $1.35(0.62-2.96)$ & $1.50(0.76-2.94)$ & $1.30(0.65-2.59)$ \\
\hline \multicolumn{7}{|c|}{ 3-h plasma glucose (mmol/l) } \\
\hline$<7.8$ & $1.38(1.01-1.89)$ & $1.26(0.91-1.75)$ & $1.32(0.96-1.82)$ & $1.32(0.95-1.83)$ & $1.26(0.93-1.71)$ & $1.12(0.82-1.54)$ \\
\hline$\geq 7.8^{\mathrm{b}}$ & $2.13(0.81-5.55)$ & $1.67(0.62-4.53)$ & $1.37(0.47-3.98)$ & $1.30(0.44-3.84)$ & $2.58(1.06-6.27)$ & $2.16(0.86-5.42)$ \\
\hline
\end{tabular}

Macrosomia is infant birthweight $>4,500 \mathrm{~g}$ and no fetal hydrops; hypoglycaemia is at least one plasma glucose value $<2.2 \mathrm{mmol} / 1 \mathrm{during}$ the first 3 days of life; hyperbilirubinaemia is at least one total serum bilirubin concentration $\geq 342 \mu \mathrm{mol} / 1$ was identified during the first 30 days of life and the following conditions were not present: positive direct antiglobulin test, glucose-6-phosphate dehydrogenase deficiency,

$\mathrm{ABO}$ incompatibility or Rhesus alloisoimmunisation.

${ }^{a}$ Adjusted for maternal age (<30 (reference) and 30+ years), race-ethnicity (non-Hispanic white (reference), African-American, Hispanic, Asian, other and unknown, and pre-pregnancy BMI (<20.0, 20.0-24.9 (reference), 25.0-29.9, 30.0+ and unknown)

${ }^{\mathrm{b}} \mathrm{ADA}$ threshold for that time period

in infants of women who were not non-Hispanic white. For example, the OR $(95 \% \mathrm{CI})$ for macrosomia associated with two or more glucose values meeting or exceeding the ADA thresholds [9] was $1.62(0.58-4.53)$ in non-Hispanic white and $9.70(2.63-35.83)$ in other infants. The corresponding ORs for hyperbilirubinaemia were $1.33(0.42-4.24)$ and 4.16 (1.11-15.52), respectively.

\section{Discussion}

Our findings suggest that pregnant women who do not meet the NDDG criteria [5], but who do exceed the Carpenter and Coustan [6] plasma glucose thresholds recommended by the ADA [9] for the diagnosis of GDM are at an increased risk of delivering an infant with macrosomia. There was also a strong suggestion that women exceeding the ADA thresholds are at an increased risk of delivering an infants with hypoglycaemia or hyperbilirubinaemia. The risk of these complications appears to increase with increasing numbers of glucose values exceeding the ADA thresholds.

It has been suggested that the underlying metabolic defect of GDM is the inability of the pancreatic beta cells to produce an adequate amount of insulin to overcome the increased insulin resistance induced by the hormonal changes occurring during pregnancy [14], which in turn leads to maternal hyperglycaemia, excess glucose exposure of the fetus and consequent fetal hyperinsulinaemia. Fetal hyperinsulinaemia leads to hyperplasia of fat tissue and skeletal muscle $[15,16]$, and possibly to neonatal hypoglycaemia [17]. Fetal hyperinsulinaemia also induces reduced oxygenation, which might increase fetal oxygen uptake, leading to increased erythropoiesis and neonatal hyperbilirubinaemia [18]. However, in this study the majority of cases of macrosomia, hypoglycaemia and hyperbilirubinaemia occurred in women with glucose levels below the ADA thresholds [9] (see Table 3), suggesting that other physiopathological mechanisms are also important determinants of these complications.

The glucose measurement most strongly associated with increased risk of each neonatal complication was the 1-h plasma glucose levels following the 100-g glucose challenge meeting or exceeding the ADA thresholds [9], suggesting that it may reflect the maternal metabolic defect leading to fetal hyperinsulinaemia more accurately than the other measures in the diagnostic OGTT. This finding also suggests that plasma glucose levels $1 \mathrm{~h}$ after an oral glucose load might be considered by other tests employed in the diagnosis of GDM, such as the 75-g, 2-h OGTT recom- 
mended by the WHO [19]. However, the WHO advocates only fasting and 2-h measurements.

Other studies [20-22] with limited power have examined the association between the ADA thresholds $[6,9]$ with perinatal complications among white women who did not meet the NDDG criteria [5]. Magee et al. [20] reported that among 34 women who met only the ADA criteria [9] the average percentage incidence of the composite measure of 33 possible perinatal complications was $41 \%$ higher than in women with normal screening. The Toronto Tri-Hospital Gestational Diabetes project [21] found that the proportion of infants with birthweight $>4,500 \mathrm{~g}$ was higher among 115 women who met only the ADA thresholds [9] than in 2,940 women with normal screening (6.1 vs $1.9 \%)$. Recently, Ricart et al. [22] reported that after controlling for possible confounders, the risk of macrosomia (defined as birthweight $>4,000 \mathrm{~g}$ ) was somewhat higher in 263 women who met only the ADA criteria [9] than in 6,350 women with normal screening $(\mathrm{OR}=1.45,95 \% \mathrm{CI}=0.83-2.52)$. In our multiethnic population, after controlling for confounders, women with GDM by only ADA criteria [9] had an increased risk of delivering an infant with macrosomia $(\mathrm{OR}=2.68)$.

The need to also lower the plasma glucose thresholds obtained during a 75-g, 2-h OGTT for the diagnosis of GDM was addressed in 1999 [23] when the WHO recommended also diagnosing women formerly classified as having IGT by lowering the plasma glucose cut points that had been in use since 1985 [19] as follows: fasting plasma glucose from 7.8 to $7.0 \mathrm{mmol} / \mathrm{l}$; 2-h plasma glucose from 11.1 to $7.8 \mathrm{mmol} / \mathrm{l}$. A recent randomised trial [3] of treatment of IGT during pregnancy (fasting plasma glucose $7.8 \mathrm{mmol} / 1$ and plasma glucose at 2 -h from 7.8 to $11.0 \mathrm{mmol} / \mathrm{l})$ assessed by a 75-g, 2-h OGTT according to the WHO recommendation [19] has shown that treatment reduces the risk of serious perinatal complications (such as death, shoulder dystocia, bone fracture and nerve palsy), as well as macrosomia. In that trial there were no significant differences between the intervention and routine-care groups regarding neonatal jaundice requiring phototherapy or hypoglycaemia requiring i.v. therapy (relative risk $(95 \% \mathrm{CI})) 0.93(0.63-1.37)$ and $1.42(0.87-2.32)$, respectively). However, it is possible that the sample size of the trial was not adequate to detect significant differences in these neonatal outcomes. In addition, levels of neonatal glycaemia or bilirubinaemia at which therapy was initiated were not defined by the study protocol. It is possible that infants of women assigned to the intervention (treatment for maternal hyperglycaemia) group were also more likely to receive perinatal interventions as suggested by the higher proportion of induced delivery and admission to the nursery unit observed in this group. Another trial [24] is assessing the benefit of tight glycaemic control on perinatal compli- cations among 950 women with at least two glucose levels (other than fasting levels) meeting or exceeding the ADA thresholds [9] during the 100-g OGTT. In that trial, if a substantial number of women meeting the ADA criteria [9], but not those of the NDDG [5], were to be enrolled, an analysis restricted to these women would help in gauging the size of the benefit of treating pregnant women with relatively mild hyperglycaemia.

Despite the fact that we had approximately 500 cases of each of the three study outcomes, analyses based on demographic subgroups had a limited ability to identify differences among the subgroups in the predictive ability of the ADA thresholds [9]. The heterogeneity we did identify (e.g. a particularly strong association between the ADA thresholds [9] and macrosomia among women who were not non-Hispanic white) should be interpreted with caution, given the larger number of subgroups we considered. It should be noted that the race-ethnicity distribution differed between each case group relative to controls. However, each case group and the control group had an extensive number of women in each race-ethnic group, permitting control for potential confounding via regression techniques, and our adjustment for this factor revealed little confounding overall. Nevertheless, it is possible that unmeasured factors that cluster with race-ethnicity can bias results. We made a great effort to measure many factors that could plausibly confound the primary association under study, extensively modelled these factors and found little confounding.

The strengths of our observational study include the use of population-based cases and controls, the completeness of case identification, the validation of cases diagnosis, the comparable assessment of eligibility criteria and potential confounders among cases and controls. Although our study was limited to a population living in the USA, it included women from several race-ethnicity groups. This broad diversity in race-ethnicity, as well as for a host of other factors, for example, body size, suggests our findings have the potential to be generalisable to other settings or countries.

Potential limitations of this study should be noted. Because no test results were blinded, we cannot eliminate the possibility that a practitioner may have altered patient management based, for example, on the finding of one glucose value on the 100-g OGTT meeting or exceeding the NDDG thresholds [5]. However, in a sensitivity analysis that excluded the 62 women who had one glucose value meeting or exceeding the NDDG thresholds [5] the associations remained between two or more pregnancy glucose values meeting or exceeding the ADA thresholds [9] and increased risk of infants macrosomia, hypoglycaemia or hyperbilirubinaemia (ORs 3.02, 3.66 and 1.90, respectively). 
Another possible limitation of this study is that there was not universal screening for infant glycaemia and bilirubinaemia. Therefore it is possible that a small number (given the cut points used for our case definitions) of infants in the control group might have had undetected hypoglycaemia or hyperbilirubinaemia. If we could have excluded such infants from the control group the observed associations between the ADA glucose thresholds and these neonatal complications might have been stronger.

The results from our study offer support for the ADA 2000 recommendations [9] to adopt the lower plasma glucose thresholds initially proposed by Carpenter and Coustan [6] for the diagnosis of GDM. These results make more pressing the need for an adequate evaluation of the impact of glycaemic control in women with 'mild' gestational diabetes.

Acknowledgements This research was supported by a R01 DK 54834 from the National Institute of Diabetes and Digestive and Kidney Diseases, a grant from the American Diabetes Association and Kaiser Community Benefit research support to A. Ferrara.

Duality of interest The authors do not have any conflict of interest.

\section{References}

1. Kjos SL, Buchanan TA (1999) Gestational diabetes mellitus. N Engl J Med 341:1749-1756

2. Jovanovic L, Pettitt DJ (2001) Gestational diabetes mellitus. JAMA 286:2516-2518

3. Crowther CA, Hiller JE, Moss JR, McPhee AJ, Jeffries WS, Robinson JS (2005) Effect of treatment of gestational diabetes mellitus on pregnancy outcomes. N Engl J Med 352:2477-2486

4. O'Sullivan JB, Mahan CM (1964) Criteria for the oral glucose tolerance test in pregnancy. Diabetes 13:278-285

5. National Diabetes Data Group (1979) Classification and diagnosis of diabetes mellitus and other categories of glucose intolerance. Diabetes 28:1039-1057

6. Carpenter MW, Coustan DR (1982) Criteria for screening tests for gestational diabetes. Am J Obstet Gynecol 144:768-773

7. Sacks DA, Abu-Fadil S, Greenspoon JS, Fotheringham N (1989) Do the current standards for glucose tolerance testing in pregnancy represent a valid conversion of O'Sullivan's original criteria? Am J Obstet Gynecol 161:638-641
8. Metzger BE, Coustan DR (1998) Summary and recommendations of the fourth International workshop - conference on gestational diabetes mellitus. The Organizing Committee. Diabetes Care 21 (Suppl 2):B161-B167

9. Gestational diabetes mellitus (2000) Diabetes Care 23:S77-S79

10. Hod M, Merlob P, Friedman S, Schoenfeld A, Ovadia J (1991) Gestational diabetes mellitus. A survey of perinatal complications in the 1980s. Diabetes 40(Suppl 2):74-78

11. Krieger N (1992) Overcoming the absence of socioeconomic data in medical records: validation and application of a census-based methodology. Am J Public Health 82:703-710

12. Go AS, Hylek EM, Phillips KA et al (2001) Prevalence of diagnosed atrial fibrillation in adults: national implications for rhythm management and stroke prevention: the AnTicoagulation and risk factors in Atrial Fibrillation (ATRIA) Study. JAMA 285:2370-2375

13. Ferrara A, Kahn HS, Quesenberry C, Riley C, Hedderson MM (2004) An increase in the incidence of gestational diabetes mellitus: Northern California, 1991-2000. Obstet Gynecol 103:526-533

14. Buchanan TA (2001) Pancreatic B-cell defects in gestational diabetes: implications for the pathogenesis and prevention of type 2 diabetes. J Clin Endocrinol Metab 86:989-993

15. Pedersen J, Bohsen-Moller B, Poulsen H (1954) Blood sugar in newborn infants of diabetic mothers. Acta Endocrinol (Copenh) 15:33-52

16. Schwartz R (1990) Hyperinsulinemia and macrosomia (editorial; comment). N Engl J Med 323:340-342

17. Schwartz R (1991) Neonatal hypoglycaemia. Back to basics in diagnosis and treatment. Diabetes 40(Suppl 2):71-73

18. Battaglia FC, Thureen PJ (1998) Nutrition of the fetus and the premature infant. Diabetes Care 21(Suppl 2):B70-B74

19. WHO Study Group (1985) Diabetes mellitus. WHO Tech Rep Ser 727:1-113

20. Magee MS, Walden CE, Benedetti TJ, Knopp RH (1993) Influence of diagnostic criteria on the incidence of gestational diabetes and perinatal morbidity. JAMA 269:609-615

21. Sermer M, Naylor CD, Farine D et al (1998) The Toronto trihospital gestational diabetes project. A preliminary review. Diabetes Care 21(Suppl 2):B33-B42

22. Ricart W, Lopez J, Mozas J et al (2005) Body mass index has a greater impact on pregnancy outcomes than gestational hyperglycaemia. Diabetologia 48:1736-1742

23. Definition, diagnosis and classification of diabetes mellitus and its complication (1999) Report of a WHO consultation. Part 1: diagnosis and classification of diabetes mellitus. WHO/NCD/ NCS/99.2. World Health Organization, Geneva, pp 1-59

24. Landon MB, Thom E, Spong CY et al (2002) A planned randomized clinical trial of treatment for mild gestational diabetes mellitus. J Matern Fetal Neonatal Med 11:226-231 